\title{
A system identification approach to baroreflex sensitivity estimation
}

\author{
Violeta McLoone and John V. Ringwood \\ Department of Electronic Engineering \\ National University of Ireland Maynooth, \\ IRELAND
}

E-mail: vmcloone@eeng.nuim.ie

jringwood@eeng.nuim.ie

\begin{abstract}
The body contains a bewildering array of regulatory systems which maintain homeostasis. There is considerable difficulty in isolating a single control loop for analysis, due to the interactions with other systems/loops. One important such regulatory loop is the baroreflex, and baroreflex sensitivity is a characteristic open-loop parameter which can help us to assess the health of the baroreflex. A diverse range of methods have been proposed to determine baroreflex sensitivity from experimental data. Unfortunately, there appears to be little consistency of result among the different methods and some explanation can be found in the nature of the problem: In most cases, an attempt is being made to determine open-loop measures from a system operating in closed-loop, subject to poor excitation. In this paper we propose a strict procedure, based on a rigourous mathematical framework, from which reliable estimates of baroreflex sensitivity can be obtained. A comparison with other methods for baroreflex sensitivity estimation, using the EuroBaVar data set, is performed.
\end{abstract}

Keywords - Baroreflex sensitivity, system identification

\section{INTRODUCTION}

The arterial baroreflex is a negative feedback control system, which detects changes in blood pressure and attempts to minimise blood pressure fluctuations, via the heart (stroke volume and heart rate) and the peripheral resistance. The baroreflex operates in conjunction with a variety of other blood pressure regulation mechanisms, such as the hormonal system and local mechanisms, operating on a variety of timescales. Fig. 1 shows a diagram of the operation of the baroreflex. For the purposes of this study, all blood pressure regulatory mechanisms apart from the baroreflex will be represented by the disturbance term, $n_{s}$, in Fig.1.

Baroreflex sensitivity (BRS) is broadly defined as the sensitivity of a change in $R$ - $R$ interval (denoted $R R$ ) to a change in arterial pressure (usually systolic arterial pressure (SAP)). BRS has considerable potential as a diagnostic tool, as baroreflex impairment has been associated with a number of conditions such as hypertension, myocardial infarction and heart failure $[1,2]$.

A large number of different methods for estimat-

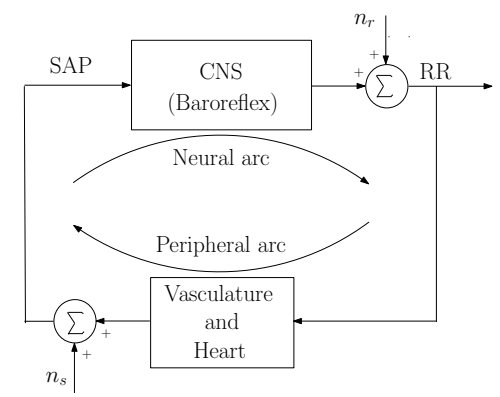

Fig. 1: Closed loop control of blood pressure via the baroreflex

ing BRS have been developed, however a 'goldstandard' technique has not been identified, and as a consequence a difficulty arises in defining what BRS values can be considered normal. Due to the lack of a 'gold standard' technique, it can be problematic to evaluate the quality of the existing BRS estimation methods, which have been summarised in section II.

This study develops a rigourous, system identification (SID) approach to estimating baroreflex sensitivity, using the EuroBaVar data set [1]. The data set comprises spontaneous recordings of $\mathrm{RR}$ 
interval and blood pressure measurements for 21 subjects in both standing and supine positions. Of the 21 subjects, one subject was a diabetic patient with evident cardiac autonomic neuropathy, and one subject was a patient who recently underwent heart transplantation. Both of these exceptional patients were considered to have effective baroreflex failure and should provide a good test for any BRS estimation technique.

\section{ApproAches to BRS DETERMINATION}

Mathematical approaches to BRS estimation from experimental data fall broadly into (a) time domain and (b) frequency domain methods. In both cases, a variety of experimental protocols are used, including both situations where external excitation (bolus injection of vasoactive substances, Valsalva manoeuvre, etc) was imposed and situations without any external stimulus. All methods use closedloop data and assume a linear relationship between $S A P$ and $R R$.

\section{a) Time-domain methods}

In some time domain methods, referred to as sequence methods, $R R$ and (usually) $S A P$ are recorded and are pre-processed into 'sequences' of concurrently increasing (or decreasing) transitions in $S A P$ and $R R[3,4,5]$. Generally, linear regression is applied to contiguous sequences and a BRS estimate is obtained by averaging the slopes of all valid sequences. Insufficient correlation between the input and output points in a sequence, low number of points in a sequence, or insufficient number of sequences can all be a major problem, especially in patients with cardiac disease [6].

A number of studies use an external stimulus to provide some excitation of the baroreflex loop, such as injection of vasoactive drugs, neck suction [7] or Valsalva manoeuvre [6]. A simple linear regression (using all of the data, without selection into sequences) is then used to estimate BRS. A major issue with such stimulations is that other blood pressure control systems are also perturbed and so it is difficult to isolate the effect of the barorelex

Some researchers utilise more complex linear time series models other than a simple regression gain $[8,9,10]$.

A nonlinear Volterra-Wiener model has also been used [11], however it is questionable [9] whether the added complexity of a non-linearity is justifiable, considering that subjects in the experimental data are at rest. Under such conditions, small signal variation requirements for local linearisation are usually met.

A Z-coefficient statistical method has also been proposed [12], but the method needs long recording periods to reliably estimate the required probabil- ity functions.

Baselli et al [13] use a closed-loop model to represent the relationship between $R R, S A P$ and respiration $(R E S P)$, while in another approach which respects the closed-loop nature of the baroreflex, bivariate autoregressive modelling has been employed to calculate the transfer functions between the signals on the feed-forward and feed-back side of the loop simultaneously [14]. A number of researchers $[15,16,17]$ take this approach to evaluate model components in the time domain and subsequently calculate the frequency response of these components.

\section{b) Frequency-domain methods}

Two main frequency bands considered for baroreflex operation are the low-frequency (LF) band of $0.04-0.15 \mathrm{~Hz}$, associated with sympathetic and parasympathetic activity and the high-frequency (HF) band of $0.15-0.4 \mathrm{~Hz}$, associated with vagal parasympathetic activity related mainly to respiration [18].

A transfer function (TF) method for BRS estimation [19] involves the calculation of the magnitude of the TF between $S A P$ and $R R$ in the LF and $\mathrm{HF}$ bands, using spectral techniques. Usually only sections of the TF with high coherence values between input and output are taken into account for the BRS estimation. The frequency-dependent gain quantity, $\alpha(f)$, defined in Eqn. (1), has also been used [20] as an estimate of BRS:

$\alpha(f)=\sqrt{\frac{G_{R}(f)}{G_{S}(f)}}$

where $G_{R}(f)$ and $G_{S}(f)$ are the spectral densities of $R R$ and $S A P$ respectively.

\section{SID FRAMEWORK FOR BRS ESTIMATION}

In attempting to estimate BRS, the characteristics of the system and the experimental protocols need to be examined, with respect to the identification problem.

- In the EuroBaVar data set [1], used in this study, spontaneous data is collected with no external excitation, which leads to $S A P$ and $R R$ having relatively small variations around their equilibrium values. Hence, a choice of a linear mathematical model would be reasonable.

- There may be relatively poor excitation of the baroreflex due to the lack of external stimulus. This may affect the identifiability of the system.

- The data available is on a 'per beat' basis i.e. a measurement of $S A P$ and $R R$ is provided 
after each RR-interval, so the data is on a non-uniform time base. This has important implications for any frequency-domain calculations.

- Data was recorded with the baroreflex intact, implying that data is collected under closedloop conditions. In addition, none of the external inputs shown in Fig. $1\left(n_{s}\right.$ and $\left.n_{r}\right)$ can be measured and there is no SAP setpoint, or desired value.

Ljung [21] identifies a framework within which a SID problem can be placed from a mathematical perspective, given the conditions, under which the data were recorded, and the nature of the data itself.

\section{a) Families of mathematical models}

Though more complex models are available [21], the most general mathematical model that we will employ is an ARMAX model (autoregressive moving average with extogenous input) given by Eq. (2):

$$
\begin{aligned}
y(k)= & \frac{B_{1}(q)}{A(q)} u_{1}\left(k-n_{d 1}\right)+\ldots \\
& +\frac{B_{m}(q)}{A(q)} u_{m}\left(k-n_{d m}\right)+\frac{C(q)}{A(q)} e(k)
\end{aligned}
$$

where $B_{i}(q), A(q)$ and $C(q)$ are polynomials of order $n_{b i}, n_{a}$ and $n_{c}$ respectively in the delay operator $q$, and $n_{d i}$ (where $\tau_{n_{d i}}=n_{d i} \Delta T$ ) is the number of pure steps delay between each of the inputs and the output. The system is, in general, a multi-input, single-output (MISO) system.

\section{b) Persistence of excitation}

Here, we identify the conditions on the input signal, $u(k)$, which allow the parameters of a mathematical model to be uniquely determined. In general, if the spectrum, $\Phi_{u}(\omega)$, of $u(k)$ is different from zero at at least $n$ points, a model of order $n$ can be uniquely identified. A convenient mathematical formulation, involving the covariance $m a-$ trix, $\bar{R}$, of $u(k)$ can be made, via:

$\bar{R}=\left(\begin{array}{cccc}R_{u}(0) & R_{u}(1) & \ldots & R_{u}(n-1) \\ R_{u}(1) & R_{u}(0) & \ldots & R_{u}(n-2) \\ \vdots & \vdots & \ddots & \vdots \\ R_{u}(n-1) & R_{u}(n-2) & \ldots & R_{u}(0)\end{array}\right)$

where:

$R_{u}(\tau)=E[u(k) u(k-\tau)]$

is defined as the covariance of $u(k), \tau$ is an integer, while $E[]$ is the expectation (averaging) operator. If $\bar{R}$ is non-singular, then $u(k)$ is persistently exciting of order $n$ and a model of order $n$ can be

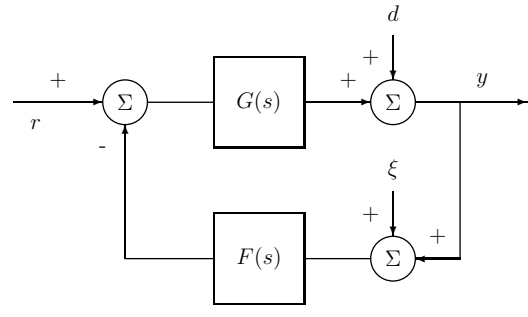

Fig. 2: Closed-loop system

uniquely identified. If $\bar{R}$ is singular, the number of non-zero singular values (i.e. the rank of $\bar{R}$ ) determines the order of the model that can be identified.

\section{c) Identification in closed-loop}

An overview of the SID framework is presented in reference to the regulatory loop of Fig.2, where $r$ is a reference (setpoint) input, $y$ is the system output, $d$ is an unmeasurable disturbance and $\xi$ is measurement noise.

In general, a number of basic conditions need to be met before identification of a system in closed loop may be attempted:

- There needs to be a delay in either the forward path $G(s)$ or the feedback path $F(s)$.

- The closed-loop system needs to be stable

- The model set proposed for identification needs to contain the true system

Three broad classes of methods for identification of dynamical systems in closed loop are available: the direct, indirect and joint input-output methods. In the direct method, used here, a prediction error method (PEM) (see Section d)) is applied directly as if no feedback exists. The signals used are the input $u(t)$ and output $y(t)$, while the reference signal $r(t)$ is ignored, even if it is known. The system is treated similarly to an open-loop system. ARX (autoregressive with exogenous input) and ARMAX models are well suited to this method, but good knowledge of the noise dynamics is necessary.

\section{d) Model parameter determination}

Given a suitable model structure, an algorithm must be employed to determine the parameters of the polynomials $A(q), B(q)$ and $C(q)$. Following the requirements for identification in closed loop the ARMAX model is formulated in prediction form as:

$\hat{y}(k \mid \Theta)=\frac{B(q)}{C(q)} u(k)+\left[1-\frac{A(q)}{C(q)}\right] y(k)$

where the hat $\left(^{\wedge}\right)$ denotes an estimate and the dependence on the parameterisation defined by $n_{a}$, 
$n_{b}, n_{c}$ and $n_{d}$ is made explicit, with:

$\Theta=\left[\begin{array}{lllllllll}a_{1} & \ldots & a_{n_{a}} & b_{0} & \ldots & b_{n_{b}} & c_{0} & \ldots & c_{n_{c}}\end{array}\right]$

We can now define the prediction error, $\varepsilon(k)$, as:

$$
\varepsilon(k, \Theta)=y(k)-\hat{y}(k \mid \Theta)
$$

A recursive optimisation algorithm is now employed to minimise the performance function:

$J(\Theta)=\frac{1}{N} \sum_{k=1}^{N} \varepsilon(k, \Theta)^{2}$

over $N$ available input/output pairs. A variety of iterative estimation algorithms may be used to determine the optimal parameter set [22].

\section{Results}

\section{a) Data preprocessing}

The $R R$ and $S A P$ data are interpolated onto a regular time axis, using a fixed sampling interval of $T=2 / 3$ secs., which is close to the average $R R$ interval. In addition, in order to focus the identification process on the frequency bands of interest, the data is filtered using a $7^{\text {th }}$ order Butterworth bandpass filter. The data is filtered both forwards and backwards, in order to achieve zero phase distortion, giving an effective filter order of 14 .

\section{b) Model order and parameter determination}

We will adopt an ARMAX model structure to specify the relationship between SAP and RR interval. The model is single input, single output, with a coloured noise term specified by $e(k)$ and the $C$ polynomial.

Following the specification in Section b), the persistence of excitation is estimated up to a potential order of 50 by evaluating the singular values of a 50x50 covariance matrix. In general, the indication is that models of order 10 and marginally greater (up to 12) can be identified with the level of excitation in the $S A P$ signal. By way of example, the singular values of the covariance matrix for Subject 5 in a supine position are shown in Fig.3. Clearly, the singular values up to order 15 are significantly above the base value.

Given the diversity of the subjects used in the collection of the EuroBaVar data, we have chosen to use the optimal model structure for each subject. In order to identify a model, which is not excessively complex, we choose a performance function which has a complexity-weighting term, viz. Akaikie's Information theoretic Criterion (AIC). In general, model orders of 6 or lower were sufficient to represent the individual data sets.

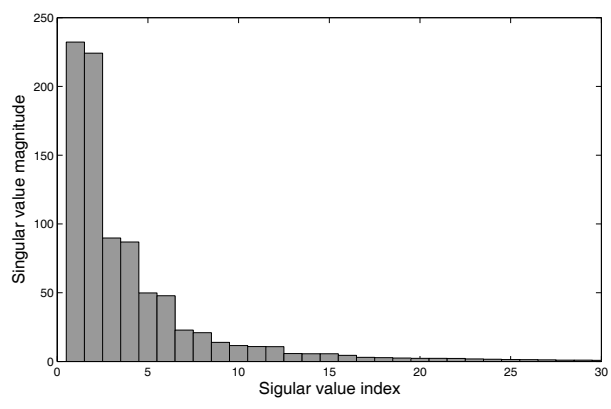

Fig. 3: Persistence of excitation in $S A P$ for Subject 5 in the supine position

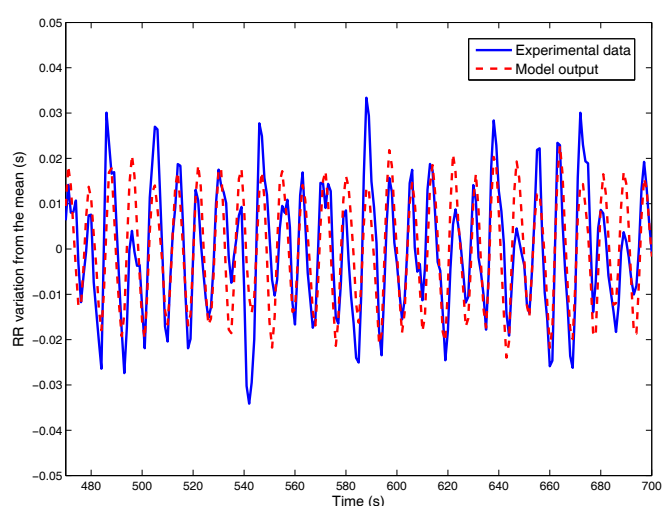

Fig. 4: Plot of actual and modelled data for Subject 5 in the supine position

As outlined in Section d), the ARMAX model parameters were determined using a prediction error method.

Figure 4 shows a comparison between the model output and the experimental data for Subject 5 in the supine position.

\section{c) Evaluation of baroreflex sensitivity}

Given that we have a time domain model for each subject, this can now be used to determine the magnitude of the frequency response across the frequency range from 0.04-0.4 Hz, containing the LF and HF baroreflex bands. We determine the magnitude response of the model from input $(u(k)=S A P(k))$ to output $(y(k)=R R(k))$ in Eq. (2) via Eq. (9):

$\left|G_{y u}(f)\right|=\left|[B(q) / A(q)]_{q=e^{2 \pi j f T}}\right|$

where $f$ is frequency in Hertz and T the sampling period of the data of $2 / 3$ secs.

Fig. 5 shows the magnitude of the frequency response for Subject 5 in the supine position. As shown in Fig.5, the baroreflex sensitivity can be calculated at the low $\left(B R S_{L F}\right)$ and high $\left(B R S_{H F}\right)$ frequency ranges by evaluating a weighted average over each range. A non-uniform Gaussian weighting function is used to determine a weighted average for the following reasons: 


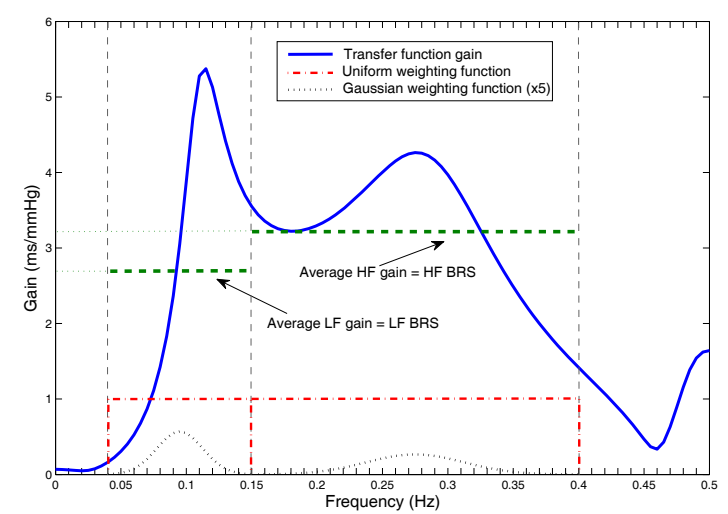

Fig. 5: Baroreflex gain for Subject 5 in the supine position

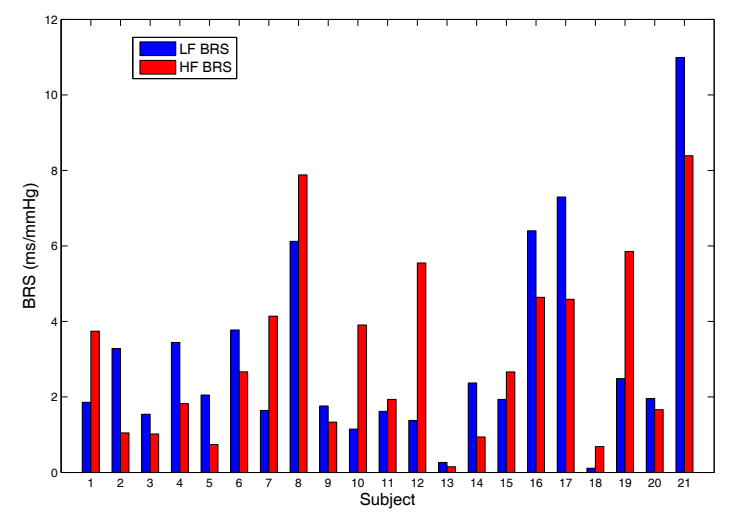

Fig. 6: BRS values for all subjects in the standing position

- The value of the baroreflex gain at the centre of the frequency range is more important than that at the fringes,

- The relatively large variation of baroreflex gain, particularly over the low frequency range (e.g. see Fig.5) renders a standard average unrepresentative of the central values, and

- Applying significant weight to the baroreflex gain at the boundary between LF and HF can result in considerable contamination between the $B R S_{L F}$ and $B R S_{H F}$ values.

Figs. 6 and 7 show the results for the BRS calculation across all EuroBaVar subjects, for the standing and supine positions respectively. In general, we note that the BRS is higher in the case of the supine position compared to standing and that $B R S_{L F}>B R S_{H F}$ for standing (12 Vs 9), while $B R S_{H F}>B R S_{L F}$ for supine (18 Vs 3 ). We can also see that subjects with impaired baroreflex (Subjects 13 and 18) are clearly identified.

Generally, a ROC (receiver operating characteristic) curve can be employed to determine what the optimum cut-off point is for detection of BRS failure. In the present study, however, there is no

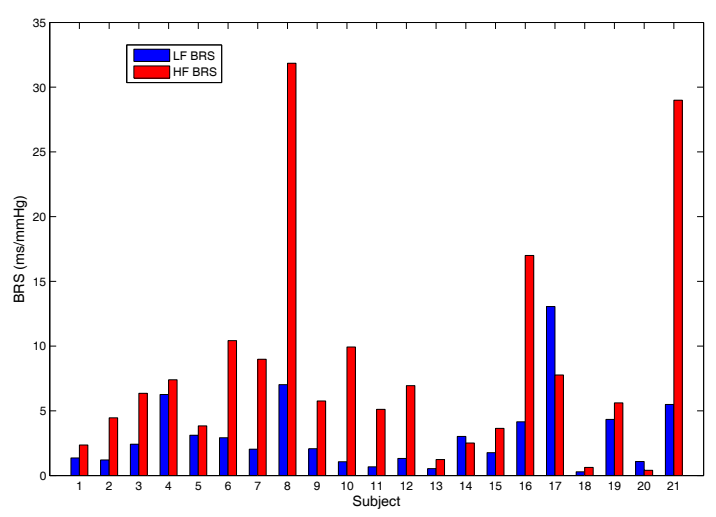

Fig. 7: BRS values for all subjects in the supine position

overlap between healthy and failed baroreflex values in each individual sets of results shown in Figures 6 and 7 .

\section{Discussion And CONClusions}

In order to assess the quality of a BRS estimate, Laude et al [1] have investigated a number of different properties of the various BRS estimation techniques used with the EuroBaVar data set.

Some of the popular estimations techniques, reported in [1], are unable to provide estimates for at least one subject, due to various thresholds not being met (minimum coherence, minimum correlation or minimum change in $\mathrm{BP}$ or $\mathrm{RR}$ interval). In general, the thresholds imposed have been designed to improve the reliability of the results; however, these conditions can lead to a restriction in the use of certain methods in practice. The procedure described in this paper does not apply specific thresholds but yields a low baroreflex gain estimate where the data set does not show a well defined relationship between SAP and RR.

BRS estimation techniques should also be able to distinguish well between data in the standing and supine positions, since BRS tends to be higher in the supine case. Our estimation technique was able to distinguish well between the two positions, however, it should be noted that the high-frequency BRS estimates showed a much better distinction between the standing and supine positions than the low-frequency estimates.

The most important feature of a BRS estimation technique is its ability to identify subjects with impaired baroreflex. Many of the BRS estimation techniques used with the EuroBaVar data set were unable to identify the two subjects with impaired baroreflex, since they were not able to provide an estimate due to threshold restrictions. The BRS estimation technique developed in this paper is well able to distinguish the two patients with baroreflex failure, as the BRS estimates are 
very low, especially in the standing position, compared to the other subjects. All the hypertensive subjects, numbered 3, 5 and 11 in Figs. 6 and 7, also show consistently lower BRS values than the healthy population. In general, the $B R S_{H F}$ index appears to have better selectivity, while BRS estimates for the standing position also appear to be able to discriminate better between healthy and impaired baroreflex, than the supine position estimates.

Our $B R S_{L F}$ estimates are well correlated with a number of other estimates reported in [1], namely the transfer function and the $\alpha$-index methods, as well as the Z-coefficient statistical method and the time-domain 'XAR' model developed by Porta et al [8]. The high correlation between the frequencydomain methods employed in [1] and our results is expected since, in effect, both our method and the frequency-domain approaches calculate a dynamic relationship or transfer function between the input and output signals (SAP and RR respectively).

In conclusion, the accurate and consistent determination of baroreflex sensitivity is a difficult task, due to the requirement to measure open-loop characteristics within a closed-loop structure relying exclusively on spontaneous measurements. Not only does the closed-loop provide a challenge for the identification method employed, but the feedback system also tries to reduce variability of the controlled variable (blood pressure), further reducing any natural excitation in the system. In order to address these challenges, we have proposed a rigourous procedure which can assess whether sufficient excitation is available and provide reliable estimates of open-loop properties.

\section{REFERENCES}

[1] D. Laude, J.-L. Elghozi, A. Girard, E. Bellard, M. Bouhaddi, P. Castiglioni, C. Cerutti, A. Cividjian, M. Di Rienzo, J.-O. Fortrat, B. Janssen, J. Karemaker, G. Lefthériotis, G. Parati, P. Persson, A. Porta, L. Quintin, J. Regnard, H. Rüdiger, and H. Stauss. Comparison of various techniques used to estimate spontaneous baroreflex sensitivity (the eurobavar study). Am J Physiol Regul Integr Comp Physiol, 286:226-231, 2004.

[2] M. La Rovere, G. Pinna, and G. Raczak. Baroreflex sensitivity: measurement and clinical implications. Ann Noninv Electrocardiol, 13 (2):191-207, 2008.

[3] L. Davies, D. Francis, P. Jurak, T. Kara, M. Piepoli, and A. Coats. Reproducibility of methods for assessing baroreflex sensitivity in normal controls and in patients with chronic heart failure. Clin Sci, 97:515-522, 1999.

[4] A. Kardos, G. Watterich, R. de Mendeez, M. Csanady, B. Casadei, and L. Rudas. Determinants of spontaneous baroreflex sensitivity in a healthy working population. Hypertension, 37:911-916, 2001.

[5] S. Gouveia, A. Rocha, P. Van de Borne, and P. Lago. Assessing baroreflex sensitivity in the sequence technique: local versus global approach. Comput Cardiol, 32:279-282, 2005.

[6] H. Oka, S. Mochio, M. Yoshioka, M. Morita, and K. Inoue. Evaluation of baroreflex sensitivity by the se- quence method using blood pressure oscillations and R-R interval changes during deep respiration. Eur Neurol, 50:230-243, 2003.

[7] D. Eckberg and P. Sleight. Human baroreflexes in health and disease. Oxford University Press, 1992.

[8] A. Porta, G. Baselli, O. Rimoldi, A. Malliani, and M. Pagani. Assessing baroreflex gain from spontaneous variability in conscious dogs: role of causality and respiration. Am J Physiol Heart Circ Physiol, 279:2558-2567, 2000.

[9] D. Patton, J. Triedman, M. Perrott, A. Vidian, and J. Saul. Baroreflex gain: characterization using autoregressive moving average analysis. Am J Physiol Heart Circ Physiol, 39:1240-1249, 1996.

[10] M. De Cecco and A. Angrilli. Measurement of human baroreceptor reflex sensitivity by means of parametric identification. Measurement, 24:187-196, 1998.

[11] T. Wu, C. Chen, and T. Kao. Baroreflex sensitivity evaluation by Volterra Wiener model and the Laguerre expansion technique. Comput Cardiol, 35:741744, 2008.

[12] M. Ducher, J. Fauvel, M. Gustin, C. Cerutti, R. Najem, G. Cuisinaud, M. Laville, N. Pozet, and C. Paultre. A new non-invasive statistical method to assess the spontaneous cardiac baroreflex in humans. Clin Sci (Colch), 88:651-655, 1995.

[13] G. Baselli, S. Cerutti, F. Baidilni, L. Biancardi, A. Porta, M. Pagani, F. Lombardi, O. Rimoldi, R. Furlan, and A. Malliani. Model for the assessment of heart period and arterial pressure variability interactions and respiration influences. Med Biol Eng Comput, 32:143-152, 1994.

[14] H. Hytti, R. Takalo, and H. Ihalainen. Tutorial on multivariate autoregressive modelling. J Clin Monit Comput, 20:101-108, 2006.

[15] G. Nollo, A. Porta, L. Faes, M. Del Greco, M. Disertori, and F. Ravelli. Causal linear parametric model for baroreflex gain assessment in patients with recent myocardial infarction. Am J Physiol Heart Circ Physiol, 280:1830-1839, 2001.

[16] L. Faes, G. Nollo, A. Porta, and F. Ravelli. Noninvasive assessment of baroreflex sensitivity in postMI patients by an open-loop parametric model of RR-systolic pressure interactions. Comput Cardiol, 26:217-220, 1999.

[17] M. Pitzalis, F. Mastropasqua, F. Massari, A. Passantino, R. Colombo, A. Mannarini, C. Forleo, and P. Rizzon. Effect of respiratory rate on the relationships between $\mathrm{RR}$ interval and systolic blood pressure fluctuations: a frequency-dependent phenomenon. Cardiovasc Res, 38:332-339, 1998.

[18] A. Malliani, F. Lombardi, and M. Pagani. Power spectrum analysis of heart rate variability: a tool to explore neural regulatory mechanisms. Br Heart $J, 71: 1-2$, 1994.

[19] H. Robbe, L. Mulder, H. Ruddel, W. Langewitz, J. Veldman, and G. Mulder. Assessment of baroreceptor reflex sensitivity by means of spectral analysis. Hypertension, 10:538-543, 1987.

[20] M. Pagani, V. Somers, R. Furlan, S. Dell'Orto, J. Conway, G. Baselli, S. Cerutti, P. Sleight, and A. Malliani. Changes in autonomic regulation induced by physical training in mild hypertension. Hypertension, 12:600610, 1988.

[21] L. Ljung. System identification: Theory for the user (2nd Ed.). Prentice-Hall, 1999.

[22] J.E. Dennis Jr. and R.B. Schnabel. Numerical Methods for Unconstrained Optimization and Nonlinear Equations. Prentice Hall, 1983. 